\title{
Upregulation of SMAD4 by MZF1 inhibits migration of human gastric cancer cells
}

\author{
JIN-HEE LEE ${ }^{1}$, SUNG-SU KIM ${ }^{1}$, HUN SEOK LEE ${ }^{1,4}$, SUNGYOUL HONG ${ }^{1}$, \\ NIRMAL RAJASEKARAN ${ }^{1}$, LI-HUI WANG ${ }^{5}$, JOON-SEOK CHOI ${ }^{6}$ and YOUNG KEE SHIN ${ }^{1-3}$ \\ ${ }^{1}$ Research Institute of Pharmaceutical Science, Department of Pharmacy, College of Pharmacy, Seoul National \\ University, Seoul; ${ }^{2}$ The Center for Anti-cancer Companion Diagnostics, Institutes of Entrepreneurial BioConvergence, \\ Seoul National University, Seoul; ${ }^{3}$ Tumor Microenvironment Global Core Research Center, Seoul National University, \\ Seoul; ${ }^{4}$ Technical Research Center, Genobio, Seoul, Republic of Korea; ${ }^{5}$ Department of Pharmacology, School of Life \\ Science and Biopharmaceutics, Shenyang Pharmaceutical University, Shenyang, Liaoning, P.R. China; ${ }^{6}$ Department \\ of Pharmaceutical Microbiology, College of Pharmacy, Catholic University, Daegu, Republic of Korea
}

Received September 12, 2016; Accepted November 24, 2016

DOI: 10.3892/ijo.2016.3793

\begin{abstract}
SMAD4 is a tumor suppressor that is frequently inactivated in many types of cancer. The role of abnormal expression of SMAD4 has been reported in developmental processes and the progression of various human cancers. The expression level of SMAD4 has been related to the survival rate in gastric cancer patients. However, the molecular mechanism underlying transcriptional regulation of SMAD4 remains largely unknown. In the present study, we characterized the promoter region of SMAD4 and identified myeloid zinc finger 1 (MZF1), as a putative transcription factor. MZF1 directly bound to a core region of the SMAD4 promoter and stimulated transcriptional activity. We also found that the expression of MZF1 influences the migration ability of gastric adenocarcinoma cells. Collectively, our results showed that MZF1 has a role in cellular migration of gastric cancer cells via promoting an increase in intracellular SMAD4 levels. This study might provide new evidence for the molecular basis of the tumor suppressive effect of the MZF1-SMAD4 axis, a new therapeutic target in advanced human gastric cancer.
\end{abstract}

Correspondence to: Professor Joon-Seok Choi, Department of Pharmaceutical Microbiology, College of Pharmacy, Catholic University, Hayang-ro 13-13, Kyeongsan-si, Daegu 38430, Republic of Korea

E-mail: joonschoi@naver.com

Professor Young Kee Shin, Laboratory of Molecular Pathology, Department of Pharmacy, College of Pharmacy, Seoul National University, 599 Gwanak-ro, Gwanak-gu, Seoul 151-742, Republic of Korea

E-mail: ykeeshin@snu.ac.kr

Key words: myeloid zinc finger 1, SMAD4, migration, gastric cancer

\section{Introduction}

SMAD4, a key regulator of TGF- $\beta$ signaling, has a critical role in cell growth, differentiation, migration and apoptosis. Initially, SMAD4 was identified as a tumor suppressor gene at a homozygous deleted region on human chromosome 18q21.1 in pancreatic ductal adenocarcinoma (1). SMAD4 inactivation at the gene or protein expression level has been shown to be essential for the progression of various tumors (2-7). It is well known that SMAD4 functional inactivation occurs by loss of heterozygosity (LOH) $(6,8-10)$, gene mutation (11-13), promoter hypermethylation (14), ubiquitin-mediated degradation (15-17) and blocking nucleo-cytoplasmic shuttling in many types of cancer (18-20). Many studies have shown that the LOH or mutations of SMAD4 are associated with a poor prognosis in advanced gastric cancer patients (21-23). We have also reported that SMAD4 expression is frequently downregulated in human gastric cancer by SMAD4 LOH or partial promoter methylation, and its alteration correlates with gastric cancer progression (24). However, this does not seem to be enough to explain the functional loss of SMAD4. Our results suggested a possibility of the downregulation of SMAD4 being affected by another mechanism. Recent studies have analyzed the genetic structure and function of the SMAD4 promoter region, and predicted several interacting transcription factors, including SP1, ETS1, NRF1 and HSF1 (25). These results propose a strong potential that SMAD4 expression is regulated by positive or negative transcription factors binding at the SMAD4 promoter region. However, the function of transcription factors for SMAD4 transcriptional activation is still not completely understood.

Myeloid zinc finger 1 (MZF1/MZF1A/MZF1B/ZNF42) is a member of SCAN-zinc finger (SCAN-ZF) transcription factor family and has been mentioned in a number of cancers and cellular functions. MZF1 is a bi-functional transcription factor that can act as both a transcriptional repressor and activator, and is involved in cellular differentiation, proliferation, migration and apoptosis in various types of cancer (26). The 
mechanism of how MZF1 is involved in cancer development, including its target molecules, is still elusive. For example, MZF1 reduces tumor invasiveness through transcriptional suppression of MMP2 (27) and IGF1R (28-30) and transcriptional activation of TNFRSF10B (DR5) (31) and FPN (ferroportin) (32) in human solid tumors. Moreover, MZF1 interacts with the tumor suppressor LDOC1 and enhances its apoptotic activity (33). However, several reports have demonstrated that overexpression of MZF1 increases proliferation, migration, and metastasis through regulation of its diverse target genes in cancer cells (34-36). Therefore, there needs to be future work carried out that clarifies and confirms the role of MZF1 in cancer.

In the present study, we identified MZF1 as a putative transcription factor of $S M A D 4$, and found that the transcriptional level of SMAD4 is increased by MZF1. In addition, we showed that MZF1 overexpression inhibits the migration of gastric cancer cells, highlighting SMAD4 as a new target for the tumor migration suppressor effect of MZF1 in gastric cancer cells, and the present study suggests potential reasons for SMAD4 transcriptional repression during tumor progression. Furthermore, our result support the notion that the MZF1-SMAD4 axis signaling mechanism could be a potential target in the treatment of gastric cancer.

\section{Materials and methods}

Cell culture. Human gastric carcinoma cells were obtained from the Korean Cell Line Bank (KCLB; Seoul, Korea). All cell lines were authenticated by short tandem repeat (STR) analysis at the characterized cell line core facility at Abion, Inc., (Seoul, Korea) during the study. The gastric carcinoma cells were maintained in RPMI-1640 medium (HyClone Laboratories, Inc., Logan, UT, USA), supplemented with $10 \%$ fetal bovine serum (FBS; HyClone Laboratories) and $1 \%$ penicillin/streptomycin (HyClone Laboratories). Cells were grown in a humidified atmosphere with $5 \% \mathrm{CO}_{2}$ at $37^{\circ} \mathrm{C}$ and routinely tested for mycoplasma infection using $\mathrm{Myco}$ VALiD Mycoplasma PCR detection kit (Intron Biotechnology, Gyeonggi-do, Korea).

Expression plasmid, si-RNA and transfection. Cells were transfected with MZF1 expression plasmid using FuGENE HD transfection reagent (Promega, Madison, WI, USA) and then incubated for $24 \mathrm{~h}$. si-SMAD4 duplexes were synthesized by Invitrogen using the following sequence: 5'-GGU CAG CCA GCU ACU UAC CAU CAU A-3'. si-MZF1 duplexes were synthesized by Cosmo Genetech Co., Ltd., (Seoul, Korea) using the following sequence: 5'-CUA CUG UAG GUG UCC AAU A-3') (35). Co-transfection of siRNA and plasmid was performed using Lipofectamine 2000 (Invitrogen, Carlsbad, CA, USA) according to the manufacturer's instruction.

Gene constructs. Luciferase reporter constructs were cloned using the restriction map of the BAC729G3 bacterial artificial chromosome (BAC) clone from the RPCI-11 human BAC library (Invitrogen), which covers the alternative promoter region of SMAD4. The $-1752 \mathrm{bp}$ upstream region of the SMAD4 transcription start site and two intra-gene region $(+20$ to +427 and +15422 to +16746$)$ were subcloned into the
pGL3 basic vector (Promega). Deletion and mutant constructs of putative SMAD4 promoters were generated by PCR. For generation of point mutation, Pfu-DNA polymerase and DpnI were used. All constructs were confirmed by sequencing.

Luciferase assay. After $24 \mathrm{~h}$ of transfection, cells were lysed with luciferase assay buffer. Then, the luciferase activity was measured using the Dual-luciferase reporter assay system according to the manufacturer's instructions (Promega) and was followed by luminescence measurement in a GENios Pro microplate reader (Tecan Trading AG, Mannedorf, Switzerland).

Electrophoretic mobility shift assay (EMSA) and chromatin immunoprecipitation (ChIP). Nuclear extract of MKN74 cells were prepared by Qproteome Nuclear Protein kit (Qiagen, Hilden, Germany) and quantified by Pierce BCA assay kit (Thermo Fisher Scientific, Waltham, MA, USA). DNA mobility shift assays were performed with the following double-stranded oligonucleotides: MZF1 5'-CTC GGA GCG GGA GGC GGG GGC AGC CGG GAG AAA GG-3'. Two complementary oligonucleotides (1000 pmol of each) were annealed and 5 pmol of the annealed oligonucleotides were 5 '-end-labeled with $\left[\gamma_{-}{ }^{32} \mathrm{P}\right]$-ATP (Amersham Biosciences, Uppsala, Sweden) using $\mathrm{T}_{4}$ polynucleotide kinase ( $\mathrm{New}$ England Biolabs, Inc., Ipswich, MA, USA). Labeled products were purified on a Sephadex G-25 column (Amersham Biosciences). For antibody supershift analysis, nuclear extracts were incubated for $30 \mathrm{~min}$ in binding buffer containing $7.5 \%$ glycerol, $15 \mathrm{mM}$ Tris- $\mathrm{HCl}, \mathrm{pH} 7.5,75 \mathrm{mM} \mathrm{NaCl}, 1.5 \mathrm{mM}$ EDTA, pH 8.0, $1.5 \mathrm{mM}$ dithiothreitol, 0.3\% Nonidet P-40 and $1 \mu \mathrm{g}$ of poly (dI-dC), and then with the probe for $40 \mathrm{~min}$ at $37^{\circ} \mathrm{C}$, and with $2 \mu \mathrm{g}$ of antibodies overnight at $4^{\circ} \mathrm{C}$ or $-20^{\circ} \mathrm{C}$. The primary antibodies used were as follows: His-probe (sc-8036) was purchased from Santa Cruz Biotechnology (Santa Cruz, CA, USA) and MZF1 (ab64866) was purchased from Abcam (Cambridge, UK). For the competition assays, 100-fold excess amount of unlabeled competitor was premixed with the radiolabeled probe before addition of the binding mixture. DNA-protein complexes were resolved on $6 \%$ nondenaturing PAGE gel at $250 \mathrm{~V}$ for $2.5 \mathrm{~h}$. After separation, the gels were dried and exposed to the phosphor screen. The relevant protein-DNA probe complexes were analyzed by a BAS-1500 Image Analyzer (Fujifilm, Tokyo, Japan). ChIP assays were performed using the EZ-ChIP Kit (Millipore, Bedford, MA, USA) according to the manufacturer's instructions. Immunoprecipitation was performed with MZF1 antibody or rabbit IgG antibody (Santa Cruz Biotechnology). DNA was analyzed by conventional PCR directed to specific regions of the SMAD4 promoter and were amplified using the respective forward and reverse primers: ChIP I (forward) 5'-CTCCCTCAAACAGGCCTTCGC-3' and (reverse) 5'-CAG CTT TCC TTT CTC CCG GCT-3'; ChIP II (forward) 5'-AGC CGG GAG AAA GGA AAG CTG-3' and (reverse) 5'-CCA AAC CGC TCC GTT ACC GCA-3'. PCR was performed for 30 cycles at $94^{\circ} \mathrm{C}(30 \mathrm{sec}), 60^{\circ} \mathrm{C}(30 \mathrm{sec})$ and $72^{\circ} \mathrm{C}(30 \mathrm{sec})$.

Quantitative real-time ( $R T)$ PCR. Total RNA was extracted by TRIzol (Invitrogen) and reversely transcribed to cDNA using the SuperScript II First-Strand Synthesis system (Invitrogen). 
Following cDNA synthesis, qRT-PCR was performed as described in a dual system LightCycler (Roche Diagnostics) and the expression levels of target genes relative to HPRT (control) were determined by a SYBR-Green-based comparative $\mathrm{CT}$ method (relative fold change $=2^{-\Delta \Delta \mathrm{CT}}$ ). Primers used are as follows: SMAD4 5'-TGG CCC AGG ATC AGT AGG T-3' (forward) and 5'-CAT CAA CAC CAA TTC CAG CA-3' (reverse); CTBP1 5'-ACT GCG TGA CCC TGC ACT-3' (forward) and 5'-GCC CCT TGT CTC ATC TGC-3' (reverse). All primers were purchased from Cosmo Genetech Co., Ltd. (Seoul, Korea).

Immunoblotting analysis. Whole cell lysates were prepared in RIPA buffer supplemented with protease inhibitor cocktail (Roche Diagnostics). Lysates were centrifuged at $4^{\circ} \mathrm{C}$, $15,000 \mathrm{rpm}$ for $20 \mathrm{~min}$. Equal amounts of protein samples were electrophoretically separated by SDS-PAGE and transferred to nitrocellulose membrane. Membranes were blocked in TBS-T (Tris-buffered saline with $0.05 \%$ Tween-20) containing 5\% non-fat dry milk and then incubated overnight at $4^{\circ} \mathrm{C}$ with the primary antibodies [SMAD4 (sc-7966), MZF1, GAPDH (FL-335)] diluted in the same buffer. GAPDH was used as loading control. Membranes were washed with TBS-T and incubated with horseradish peroxidase-conjugated secondary antibodies (Pierce, Rockford, IL, USA). The results were visualized with ECL reaction.

Screening analysis of transcription factor binding site (TFBS). MatInspector (Genomatix Software GmbH, Munich, Germany; http://www.genomatix.de) was used to locate regulatory elements within the aforementioned core promoter region, and the internet-based TFSEARCH: Searching TFBS program (http://www.cbrc.jp/research/db/TFSEARCH.html) was used to localize the putative transcription factor binding sites within the 5'-flanking region of SMAD4. Alignment of human and mouse promoter sequences was performed with NCBI's Ensemble interface. Furthermore, mouse and chimpanzee SMAD4 promoter sequences were compared with human genomic sequences for conservation of the MZF1 binding motif.

Cell proliferation assay. Cell proliferation assays were performed using the EZ-Cytox kit (Daeil Lab Service, Co.,Ltd., Seoul, Korea) according to the manufacturer's instructions. WST assays were performed as previously described (37). Cell were seeded at the density of $1 \times 10^{5}$ cells and transfected with the expression vector.

Cell migration assay. Cell migration was analyzed using 24-well Transwell plates with polycarbonate membranes (Corning Costar, Corning, NY, USA). For Transwell migration assay, cells were transfected with expression vectors encoding wild-type (WT)-MZF1 or si-MZF1. Cells were prepared $24 \mathrm{~h}$ post-transfection and then loaded into the upper compartment. After incubation for $24 \mathrm{~h}$ at $37^{\circ} \mathrm{C}$, the cell number was detected with a GENios Pro microplate reader (Tecan Trading AG) using $485 / 535 \mathrm{~nm}$ filter set as previously described. The migration assay was performed in at least three independent experiments. Values are expressed as percentages compared to the control. The in vitro wound-healing assay was performed to examine the migration on gastric cancer cells transfected with WT-MZF1 vectors or si-MZF1. Transfected cells were grown on 96-well plates with their respective culture media. After the growing cell layers had reached confluence, wounds were prepared by a single scratch on the monolayer using a wound maker and the wounded layers were washed with phosphatebuffered saline (PBS) to remove the cell debris. Cell plates were applied to the IncuCyte ZOOM (Essen BioScience, Inc., Ann Arbor, MI, USA) and scanned every $1 \mathrm{~h}$ for 24 or $48 \mathrm{~h}$. The wound-healing assay was performed in triplicate in at least three independent experiments.

Statistical analysis. The results were compared using one-way ANOVA analysis followed by the Turkey's test for multiple comparisons. Means were considered significant, at $\mathrm{P}<0.05$. Statistical analysis was performed using a GraphPad Prism package for personal computers (GraphPad Software, Inc., San Diego, CA, USA). Results were considered significant at $\mathrm{P}<0.05, \mathrm{P}<0.01$ or $\mathrm{P}<0.001$. All the data with error bars are presented as mean \pm SD for at least three independent experiments.

\section{Results}

MZF1 positively regulates SMAD4 promoter activity and expression. To explore the transcriptional regulator candidates of the SMAD4 promoter, we investigated the region from -1752 to +84 (1836 bp) to the transcription start site using bioinformatic tools, such as MatInspector professional software and the internet-based TFSEARCH database. In our previous study, we identified the transcriptional start site of SMAD4 by reverse transcription-PCR and nucleotide sequencing, and we reported that hypermethylation of $\mathrm{CpG}$ site within this region might be related to the transcriptional silencing of SMAD4 (37). Our previous results on the identity of the transcription start site were consistent with the European Molecular Biology Laboratory database (http://www.ensembl.org), despite of the presence of several alternative transcripts encoding exon 1 and exon 2 of SMAD4. In this analysis, we screened three transcription factors, HSF1, RUNX1 (AML-1) and MZF1, which have a higher likelihood of interaction than other transcription factor candidates (Fig. 1A). According to previous reports, we first performed a comparison analysis of reported smad4 promoter regions in HEK293T and SNU638. HEK293T cell was used as positive control (25). The basal promoter activity of these three different SMAD4 promoter regions was investigated using a vector constructs: Luc-1752 (-1752 to +84), Roth et al (38) (+20 to +427$)$ and Minami et al (39) (+15422 to 16746). As shown in Fig. 1B, luciferase activity of the -1752 to +84 (Luc-1752) construct was nearly 80 to 150 -fold increased compared with the pGL3 control, whereas the other constructs did not have effect on SMAD4 promoter activity. Our data suggested that the -1752 to +84 region is essential for basal SMAD4 promoter activity, we further concentrated on the role of this region. The transcriptional activity of these three transcription factors on the SMAD4 promoter region was investigated using a vector construct 'Luc-1752', a luciferase-conjugated SMAD4 promoter region. As shown in Fig. 1C, transient co-transfection of Luc-1752 and the MZF1 expression vector exhibited over a 2.5 -fold increase in the induction on transcriptional 

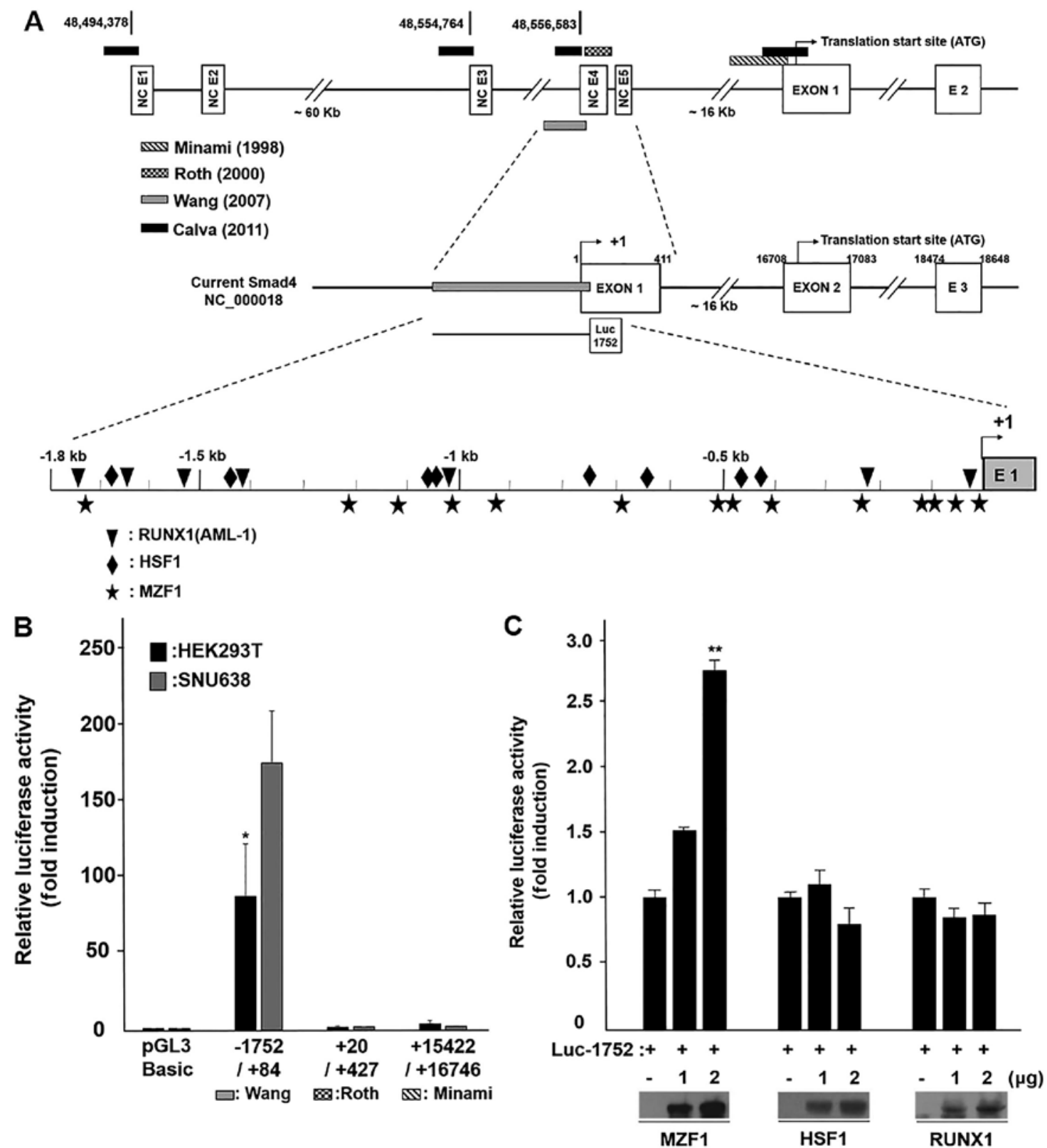

Figure 1. MZF1 positively regulates SMAD4 promoter activity and expression. (A) Schematic presentation of 5'-flanking region of human SMAD4 promoter (top line) and current SMAD4 gene structure (middle line). Black square, Calva et al (25); Dot, Roth et al (38); slash, Minami et al (39); Gray, Wang et al (24). Three transcription factors binding site locus in the SMAD4 promoter and reporter construction for luciferase assay. (B) The basal promoter activity of the three different SMAD4 promoter regions. (C) Transcription factor MZF1 activation of the SMAD4 promoter. Relative luciferase activity was normalized by the wild-type Renilla luciferase activity. Data represent the mean $\pm \mathrm{SD}$. ${ }^{*} \mathrm{P}<0.05,{ }^{* *} \mathrm{P}<0.01$ (Student's t-test).

activity of SMAD4 promoter. However, co-transfection of Luc-1752 with HSF1 or AML-1 (RUNX1) did not reveal an increase in the SMAD4 promoter activity. Next, we analyzed SMAD4 and MZF1 expression in 13 gastric cancer cell lines. We found that $S M A D 4$ mRNA $(r=0.28, \mathrm{P}=0.002)$ and SMAD4 protein $(\mathrm{r}=0.35, \mathrm{P}=0.38)$ levels showed a close correlation with MZF1 protein level (Fig. 1D). In particular, their expression was substantially decreased in KATOIII, MKN28, MKN74, NCI-N87 and SNU5 cells, whereas MKN1, SNU484, SNU620 and SNU668 had relatively high levels of their expression. In addition, ectopic expression of MZF1 increased SMAD4 levels in MKN74 cells (low expresser), whereas si-MZF1 transfection decreased SMAD4 levels in MKN1 cell (high expresser). MZF1 affects SMAD4 expression at both the
mRNA and protein levels in gastric cancer cell lines (Fig. 1E). These results suggest that SMAD4 is a novel target gene of the transcription factor MZF1.

MZF1 directly binds to MEB2 (-80 to -77) of SMAD4 promoter region. To analyze the core region enabling MZF1mediated transcriptional activation on the SMAD4 promoter, we generated four constructs, each containing a partial deletion mutant in the SMAD4 promoter, luc-1011, -371, -216 and -41 for luciferase assaying (Fig. 2A). As shown in Fig. 2A, transient co-transfection of the MZF1-expressing vector and each partial deletion mutant construct exhibited induction in SMAD4 promoter activity from 1.7- to 3 -fold. Among them, the Luc-216 construct showed the highest SMAD4 promoter 

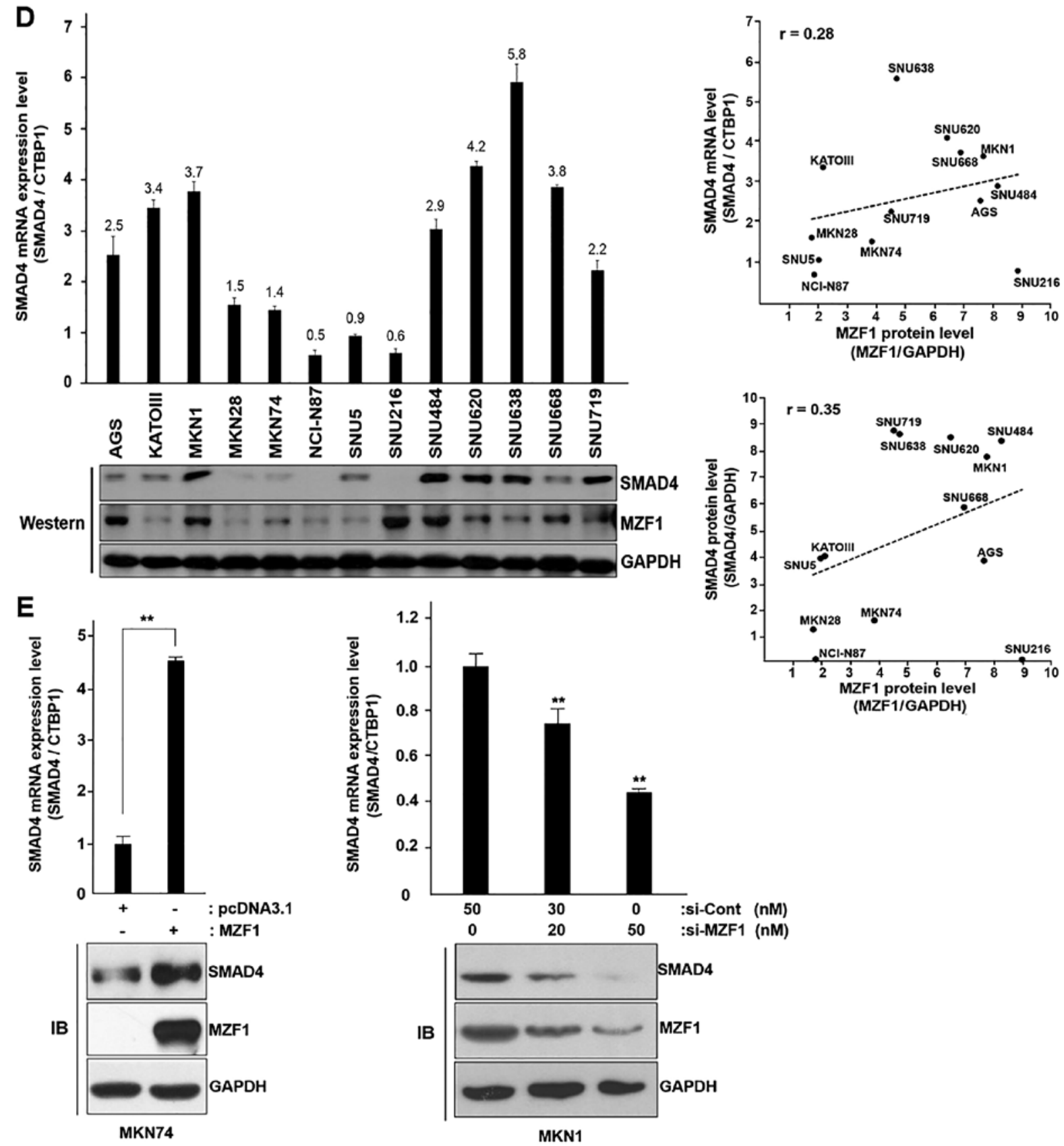

Figure 1. Continued.(D) qRT-PCR and immunoblotting assays of SMAD4 and immunoblotting assay of MZF1 in gastric cancer cell lines. SMAD4 mRNA and SMAD4 protein levels showed a close correlation with MZF1 protein level: weak correlation $(+0.1$ to +0.3$)$, clear correlation $(+0.3$ to +0.7$)$, strong correlation $(+0.7$ to +1.0$)$. (E) Cells were transfected with WT-MZF1 or si-MZF1 and SMAD4 mRNA, and protein expression was determined by qRT-PCR and immunoblotting assays. The qRT-PCR values in $\mathrm{D}$ and $\mathrm{E}$ were normalized to the housekeeping gene CTBP1. IB, immunoblot. ${ }^{*} \mathrm{P}<0.05$, ${ }^{* *} \mathrm{P}<0.01$ (Student's t-test).

activity under MZF1 overexpression. In further bioinformatic analysis, we identified four putative MZF1-binding elements (MBE) on the Luc-216-containing partially deleted SMAD4 promoter region (nucleotides -216 to +84$)$ : MBE 1 (-102 to -99$)$, MBE 2 (-80 to -77$)$, MBE 3 (-52 to -49$)$ and MBE 4 (-12 to -9$)$. To demonstrate essential MZF1-binding element, we generated mutant reporter constructs containing mutated MBE sequences (5'-AAAAG-3') that disturb MZF1 binding. According to the results (Fig. 2B, left panel), mutation of MBE2 showed loss of promoter activity, whereas other mutated MBEs exhibited over 1.5 -fold increase in induction compared to the negative control (Fig. 2B, right panel). These results show that MZF1 could positively regulate SMAD4 promoter activity and its critical binding region might be MBE2 (-80 to -77) located from -216 to +84 in the SMAD4 promoter. To examine the direct binding of MZF1 to the SMAD4 promoter region, we performed ChIP assays and EMSAs. For ChIP analysis, we produced ChIP I and ChIP II sequences containing two independent MBE sites and we also designed, an EMSA probe (Fig. 3A). In ChIP analysis, MZF1 directly bound to the ChIP I sequence containing MBE1 and MBE2 sites (Fig. 3B). Moreover, EMSA revealed that MZF1 specifically interacted with the EMSA probe containing the MBE2 site (Fig. 3C) and addition of an MZF1 antibody to the reaction mixture for EMSA resulted in a supershifted band. These results indicate that MZF1 directly bind to MEB2 (-80 to -77) of SMAD4 promoter region. 


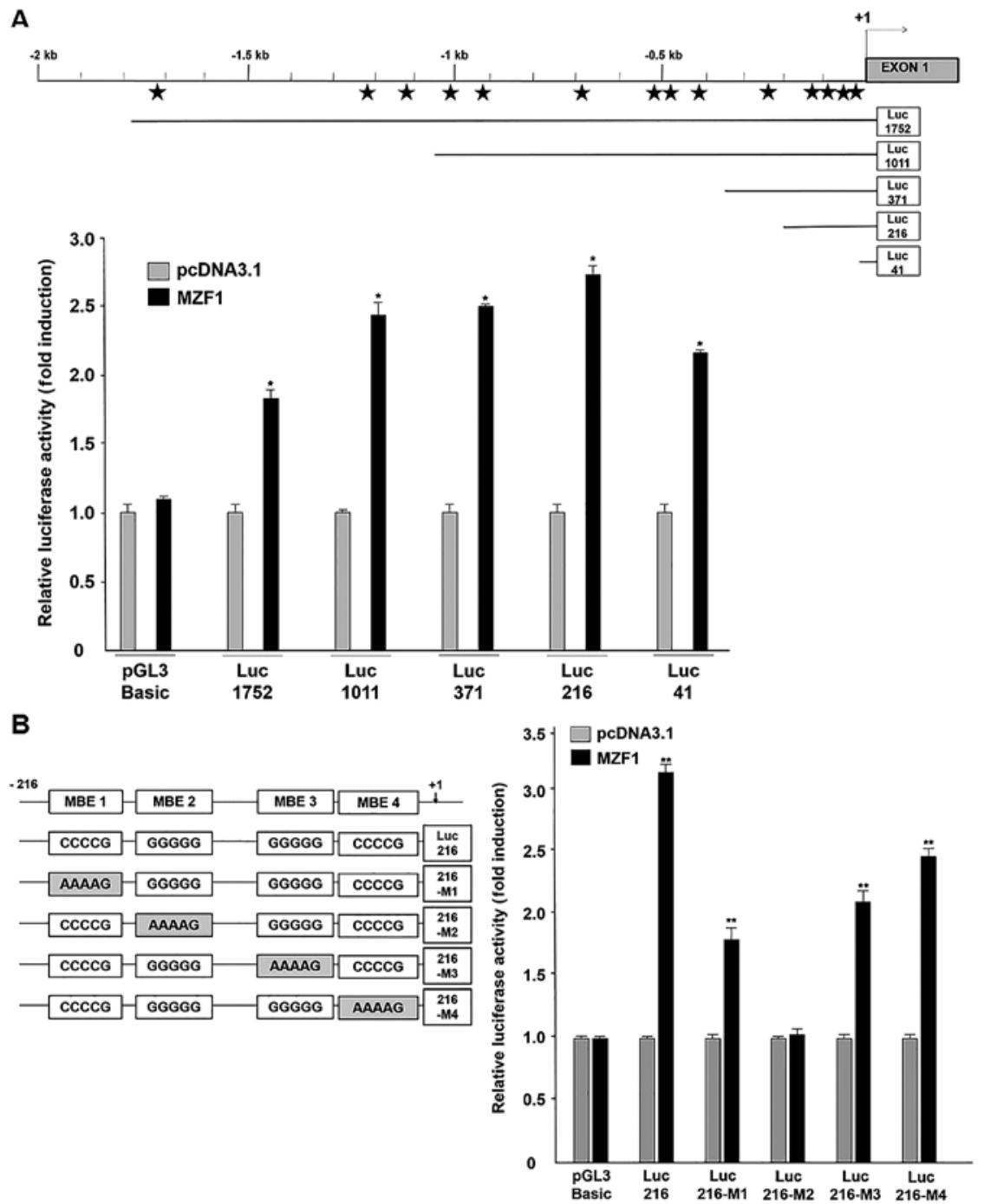

Figure 2. Identification of a critical region controlling SMAD4 expression. (A) An MZF1 binding element in the SMAD4 promoter and reporter construction for luciferase assay. Asterisk indicates MZF1 binding element. Promoter assay for SMAD4 induction by MZF1. Relative luciferase activity was normalized by the wild-type Renilla luciferase activity. Data represent the mean $\pm \mathrm{SD}$. ${ }^{*} \mathrm{P}<0.05$ (Student's $\mathrm{t}$-test). (B) Schematic presentation of MEB (MZF1 binding element) in Luc-216 construct and mutation construct. Loss of SMAD4 promoter activity of MZF1 by MEB2 site mutation. ${ }^{* *} \mathrm{P}<0.01$ (Student's t-test).

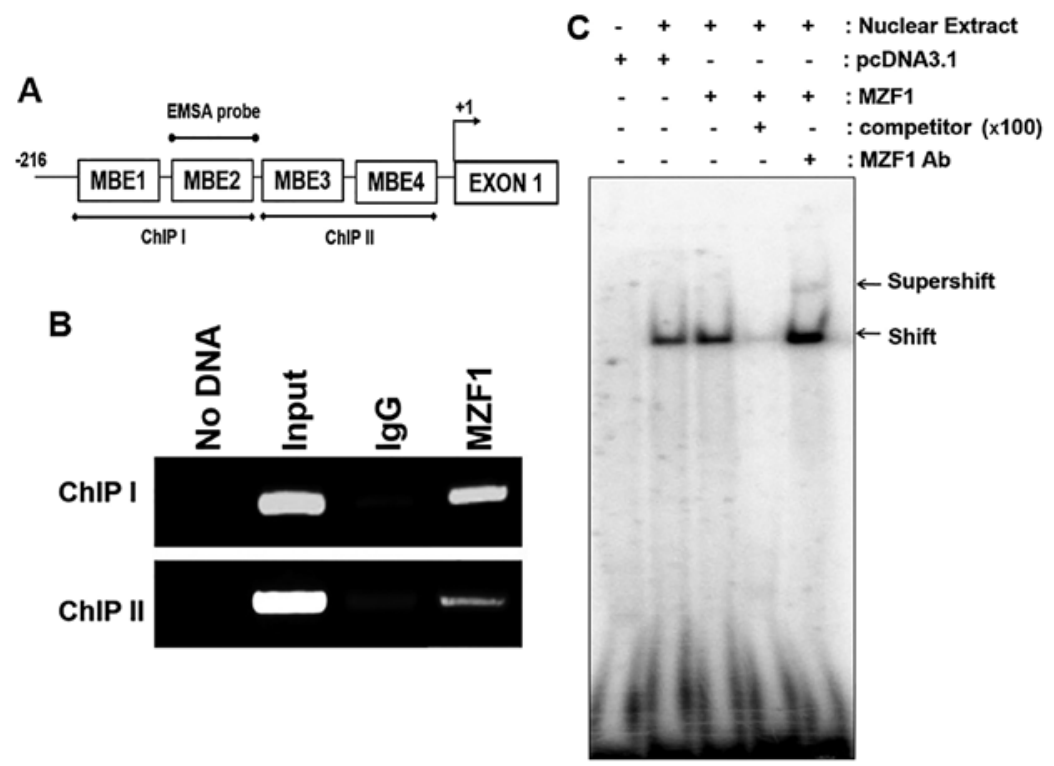

Figure 3. SMAD4 is a direct transcription target of MZF1. (A) Schematic presentation of ChIP assay regions (ChIP I and ChIP II) and EMSA probe. (B) ChIP assay for MZF1 binding to the SMAD4 promoter. No DNA, negative control. (C) EMSA for MZF1 interaction with the MEB2 site (-81 to -77). 
A

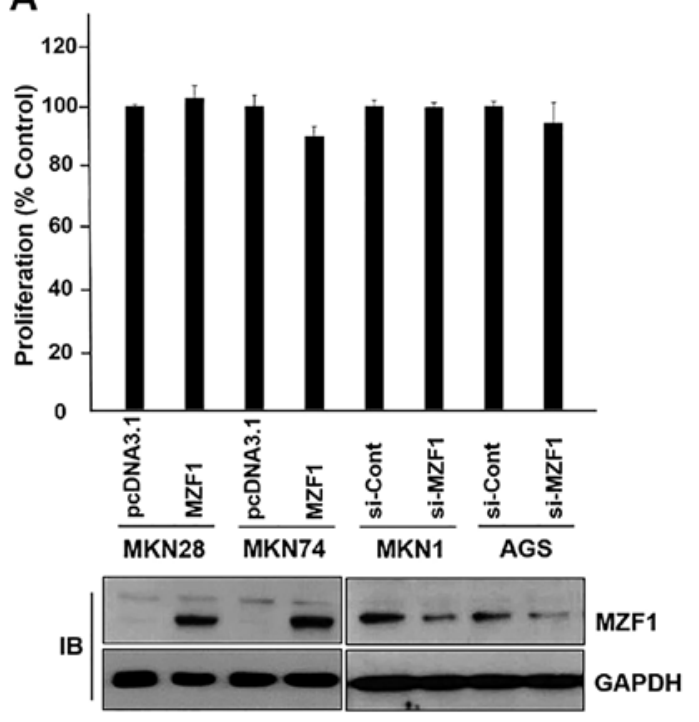

C

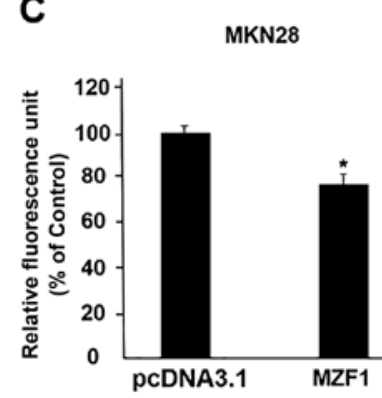

D

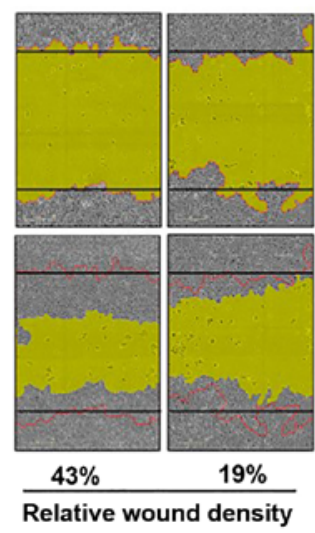

MKN74
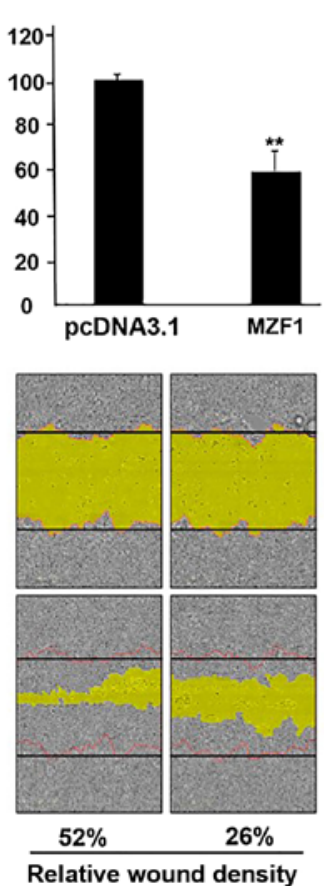

B
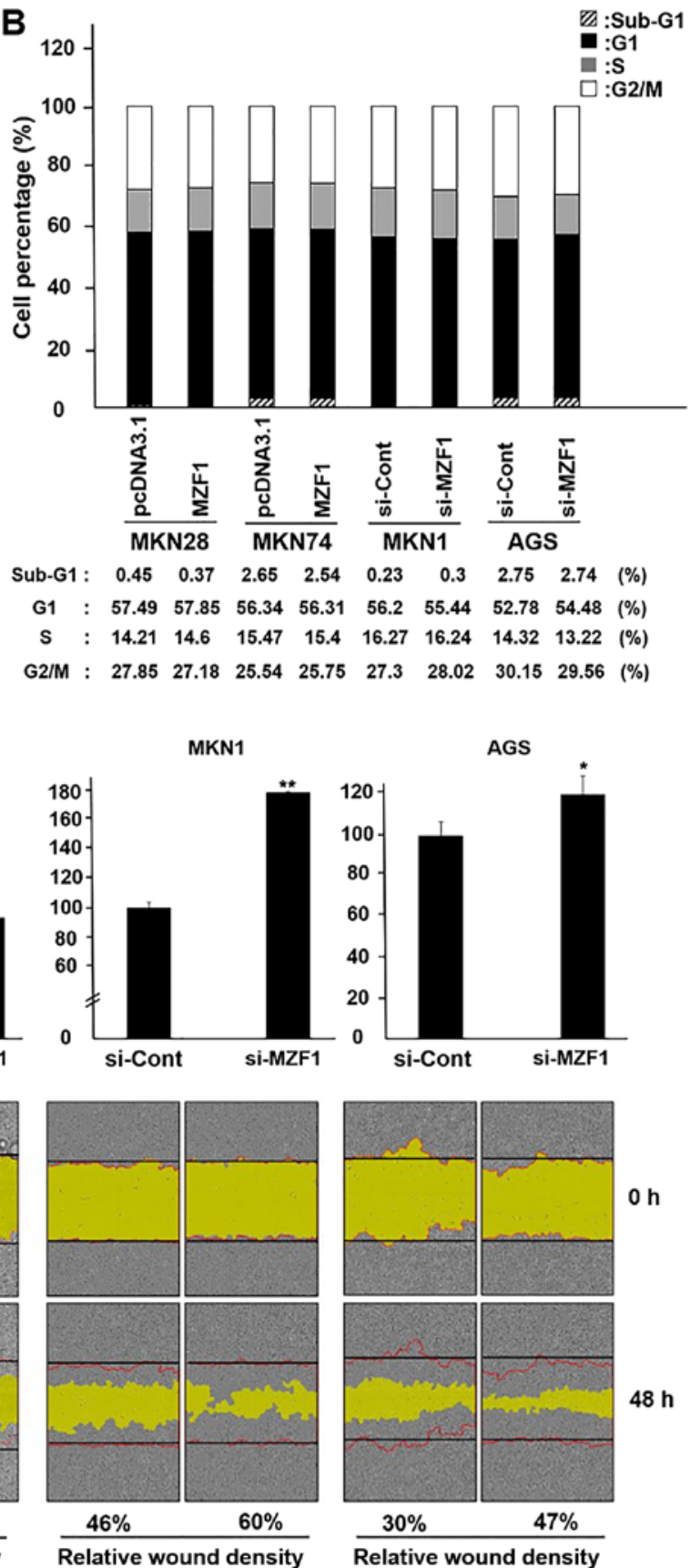

Figure 4. MZF1 inhibits gastric cancer cell migration. (A) MZF1 effect on cell proliferation. MKN28, MKN74, MKN1 and AGS cells were transfected with WT-MZF1 $(2 \mu \mathrm{g})$ or si-MZF1 $(20 \mathrm{nM})$ for $24 \mathrm{~h}$ and analyzed with WST assay. (B) No effect of MZF1 on cell cycle using FACS analysis. (C and D) Role of MZF1 in cancer cell migration. The Transwell migration assay determined cell migration in MKN28, MKN74, MKN1 and AGS cells transfected with WT-MZF1 or si-MZF1. The wound healing assay determined cell migration in MKN28, MKN74, MKN1 and AGS cells transfected with WT-MZF1 or si-MZF1. Data represent the mean $\pm \mathrm{SD} .{ }^{*} \mathrm{P}<0.05,{ }^{* *} \mathrm{P}<0.01$ (Student's t-test).

MZF1 inhibits migration of gastric cancer cells. To elucidate the molecular function of MZF1 in gastric cancer cells, we performed proliferation assay using WST reagent and cell cycle analysis by flow cytometry after overexpression or knockdown of MZF1. As shown in Fig. 4A, transient transfection of WT-MZF1 did not affect MKN28 and MKN74 cell proliferation and siRNA-mediated knockdown of endogenous MZF1 neither affected MKN1 and AGS cell proliferation, nor did it affect the cell cycle change of the 4 cell lines (Fig. 4B). Next, we investigated whether MZF1 influences migration of gastric cancer cells. We performed migration assay using the Transwell migration assay and woundhealing assay. As a result, cell migration was substantially decreased and increased by transfection of WT-MZF1 and si-MZF1 (Fig. 4C and D). These results support the idea that MZF1 overexpression might negatively regulate migration, but does not have an effect on cell proliferation and growth of gastric cancer cells.

MZF1 suppresses cancer cell migration by enhancing SMAD4 expression. According to previous reports, SMAD4 plays an important role in the regulation of cancer cell migration, 

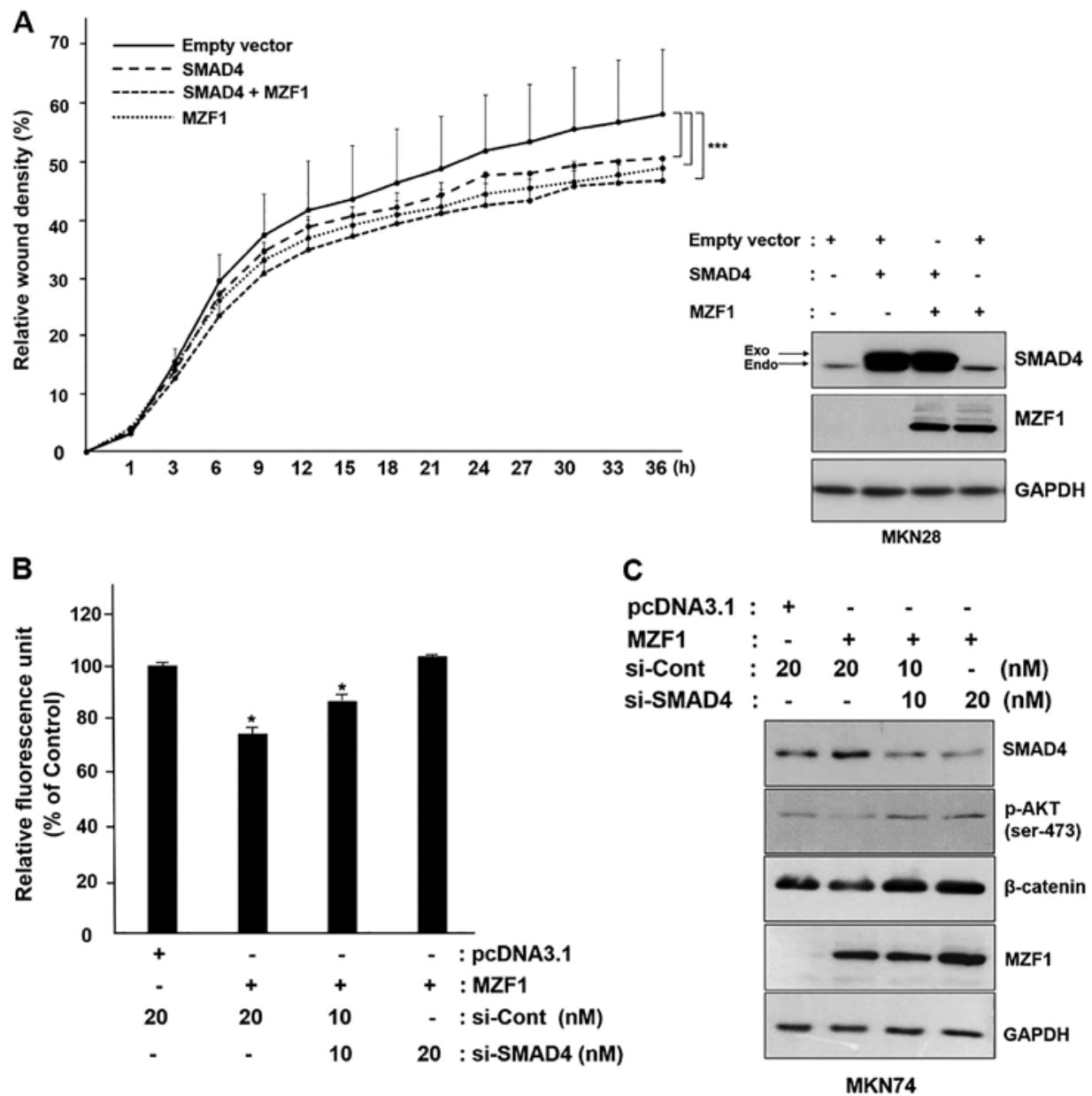

Figure 5. Requirement of functional SMAD4 for MZF1 suppresses cancer cell migration. (A) MZF1 effect on SMAD4 inhibits cancer cell migration. A wound-healing assay was performed MKN28 cells transfected with WT-SMAD4 and WT-MZF1. Data represent the mean \pm SD. ${ }^{* * *} \mathrm{P}<0.001$ (Student's t-test). (B and C) Role of SMAD4 and MZF1 in inhibiting cancer cell migration. Transwell assay and immunoblotting were performed using MKN74 cells transfected with WT-MZF1 and si-SMAD4. Data represent the mean \pm SD. "P<0.05 (Student's t-test).

and therefore, we analyzed whether MZF1 suppresses cancer cell migration through regulation of SMAD4 expression. As shown in the Fig. 5A, co-transfection of MZF1 and SMAD4 into MKN28 cells significantly exhibited suppressive effect on cellular migration in the wound-healing assay. Consistent with this, the inhibitory effect on migration by overexpression of MZF1 and SMAD4 is reinforced due to MZF1-mediated increase in the expression of SMAD4. We next examined whether silencing of SMAD4 expression inhibits MZF1mediated cellular migration in human gastric cancer MKN74 cells. Transient overexpression of MZF1 revealed over $30 \%$ reduction of MKN74 migration. In addition, co-transfection of si-SMAD4 restored this MZF1-induced reduction of migration in a dose-dependent manner (Fig. 5B). Likewise, the data from immunoblotting analysis showed that a knockdown of SMAD4 expression increased phosphorylation of AKT and expression of $\beta$-catenin, which are key regulatory molecules in migration. As would be expected, downregulation of AKT phosphorylation and suppression of $\beta$-catenin expression was observed when MZF1 was overexpressed (Fig. 5C). In summary, our findings indicated that MZF1 transcriptionally upregulates expression of SMAD4, and this MZF1-mediated expression of SMAD4 act as a suppressor of cancer migration in gastric cancer cells.

\section{Discussion}

Gastric cancer is one of the most commonly diagnosed malignancies worldwide. Many risk factors have been associated with the development of gastric cancer and the multifactorial pathogenic mechanisms including gastric adenomas, polyps, and Helicobacter pylori infection (23). SMAD4 is a multifunctional protein and its tumor suppressor effect has been reported in many studies (40-42). The loss of SMAD4 expression is an especially common feature in human gastric cancer and is a critical event in the development and progression of gastric cancer $(22,23)$.

The well-known mechanisms of SMAD4 inactivation can be divided into five groups: i) $\mathrm{LOH}$ is related to mechanisms of SMAD4 inactivation. Previous studies have demonstrated that deletion of SMAD4 frequently occurred in various cancers including those of the brain (6), lung (9), bladder (10) and colon (8). We have also reported that $S M A D 4 \mathrm{LOH}$ was detected in 20 of $70(29 \%)$ gastric cancer patients. Loss of SMAD4 expression occurred in 10 of $20(50 \%)$ LOH-positive cases. LOH of the SMAD4 locus was correlated with loss of SMAD4 mRNA and SMAD4 protein expression in gastric carcinoma and gastric cancer cells (24). ii) SMAD4 inactivation occurs by SMAD4 mutation, which is frequently associated with pancre- 
atic (13), head and neck (11) and colon (12) cancers. Mutational events of SMAD4 in various cancers were correlated with loss of SMAD4 expression, which is associated with tumor malignant progression (1). However, SMAD4 mutation is an infrequent occurrence. We have also reported that SMAD4 mutations were not found in tissue samples of gastric carcinoma (24). iii) SMAD4 reduction is associated with promoter hypermethylation. Previously, SMAD4 methylation has been studied in colon and prostate cancer. The methylation-specific PCR primers used in these studies were designed in the +20 to +427 region of the current SMAD4 transcription start site $(14,38)$. However, we were the first group to confirm the location of the SMAD4 transcription start site that is different from the previously reported SMAD4 promoter region. We have reported that SMAD4 methylation is correlated with loss of SMAD4 expression. However, SMAD4 promoter methylation was rare in gastric cancer progression (24). iv) Functional loss of SMAD4 occurs by ubiquitin-mediated degradation. For instance, SMAD4 is targeted for degradation through interactions with proteins including Jun-activating binding protein 1 (JAB1) (16), SCF $\beta$-TrCP-ubiquitin ligase complex (15), and the carboxyl-terminus of Hsc-70 interacting protein (CHIP) (17). v) SMAD4 is inactivated as a result of interference in the nucleus-cytoplasm shuttle of SMAD4. SMAD4 shuttles continuously between the cytoplasm and the nucleus (27). We have also reported that nuclear expression, and not cytoplasmic expression, is strongly correlated with the prognosis in gastric cancer (43). Nucleocytoplasmic shuttling is specific for SMAD4-induced target gene regulation and the process can involve R-SMADs. Previous studies indicated that the activation of SMAD4 nuclear export signal (NES) depends on the nuclear transport receptor CEM 1 (19). In addition, some retention factors, such as microtubules (20), or nuclear import proteins, such as ELF (embryonic liver fodrin) (18), could also be involved in the subcellular distribution of SMAD4. Taken together, these factors are highly significant for SMAD4 functional inactivation. However, this does not seem to be enough to explain the functional loss of SMAD4. Therefore, in this study, we focused on the regulation of SMAD4 expression by transcription factors and searched for new transcription factors based on the SMAD4 promoter region we previously reported (24). In the present study, we show that expression levels of MZF1 and SMAD4 are low and extremely low, respectively, in cells including KATOIII, MKN28, MKN74, NCI-N87 and SNU5. Moreover, protein expression of MZF1 is correlated with SMAD4 mRNA $(\mathrm{r}=0.28, \mathrm{P}=0.002)$ and SMAD4 protein expression $(\mathrm{r}=0.35, \mathrm{P}=0.38)$ in gastric cancer cell lines. Our previous studies showed that loss of SMAD4 expression was caused by LOH (AGS, KATOIII, MKN28, MKN74, SNU5 and SNU216), promoter methylation (NCI-N87) and mutation (SNU216). These correlations were more increased when SNU216 cell with a SMAD4 LOH and mutation were removed: SMAD4 mRNA and MZF1 protein $(\mathrm{r}=0.52, \mathrm{P}=0.003), S M A D 4$ protein and MZF1 protein $(\mathrm{r}=0.65$, $\mathrm{P}=0.28$ ). Our data suggested that SMAD4 inactivation may be caused by not only by LOH or promoter methylation but also by abnormal expression of MZF1. This suggests that MZF1 may contribute to mechanistic inactivation of SMAD4 in gastric cancers. After investigation, we found that SMAD4 expression was increased by the transcription factor MZF1 in gastric cancer cells, regulated through its direct binding to the $S M A D 4$ promoter region. This is the first study reporting a positive transcriptional regulator for SMAD4. In a previous study, it was shown that overexpression of MZF1 inhibits cancer cell migration and tumorigenesis $(27,44)$. MZF1 is a multifunctional protein and its underlying molecular mechanisms have not been fully clarified. Some studies have found that the overexpression of MZF1 inhibits apoptosis and promotes oncogenesis $(45,46)$ showing that MZF1 might function as a potential oncogene contributing to the development and progression of human cancers (34). Although the role of MZF1 in tumorigenesis is still controversial, we show that overexpression of MZF1 inhibits gastric cancer cell migration through decreased AKT and $\beta$-catenin expression. Interestingly, SMAD4 is a well-known tumor migration suppressor; it elicits its antitumor effects by blocking $\beta$-catenin signaling and SMAD4 directly suppresses the Wnt/ $\beta$-catenin signaling activity in colon cancer cells by decreasing $\beta$-catenin mRNA expression (47). In addition, the loss of SMAD4 activity activates the AKT pathway through upregulation of anti-apoptotic proteins including BCL2, BCL2L2 (BCLW) and survivin (48). Moreover, we found that SMAD4 regulated the suppression of WNT/ $\beta$-catenin signaling by downregulating the oncoprotein AURKA in cancer (49) and contributed to the tumor suppressor function of Tob1 including apoptosis and inhibiting proliferation, migration, and invasion in gastric cancer cells $(37,50)$. Considering this fact, although both MZF1 and SMAD4 have exhibited functions of migration suppression, MZF1 likely inhibits cancer cell migration in conjunction with SMAD4. In this study, we found that SMAD4 plays a critical role in MZF1-inhibited gastric cancer cell migration. The migration suppressor function of MZF1 is possible through regulation of SMAD4 expression. The present study provides new insight into the molecular basis for the tumor suppressive effect of the MZF1-SMAD4 axis in gastric cancer cells. Taken together, SMAD4 is a novel target of MZF1, which promotes the tumor suppressor function of MZF1 in gastric tumorigenesis. This finding indicates that low expression of MZF1 is linked to transcriptional repression of SMAD4, at least in gastric cancer progression. We suggest that MZF1-SMAD4 signaling may represent a new therapeutic target in advanced human gastric cancer.

\section{Acknowledgements}

The present study was supported by a grant (nos. NRF-2014R1A1A1008685 and NRF-2015R1C1A2A01055635) from the National Research Foundation of Korea (NRF) by the Ministry of Science and ICT \& Future Planning. Also, we thank Dr Tara L. Sander (Medical College of Wisconsin, Milwaukee, Wisconsin) for MZF1 (pcDNA 3.1-MZF1b-myc/ His(-)) vector.

\section{References}

1. Miyaki M and Kuroki T: Role of Smad4 (DPC4) inactivation in human cancer. Biochem Biophys Res Commun 306: 799-804, 2003.

2. Yamada S, Fujii T, Kanda M, Sugimoto H, Nomoto S and Kodera Y: Clinical significance of SMAD4 expression in resectable pancreatic cancer: Correlation with tumor progression and recurrence pattern. Cancer Res 74 (19 Suppl): 38312014. 
3. Sasaki S, Yamamoto H, Kaneto H, Ozeki I, Adachi Y, Takagi H, Matsumoto $\mathrm{T}$, Itoh $\mathrm{H}$, Nagakawa $\mathrm{T}$, Miyakawa $\mathrm{H}$, et al: Differential roles of alterations of p53, p16, and SMAD4 expression in the progression of intraductal papillary-mucinous tumors of the pancreas. Oncol Rep 10: 21-25, 2003.

4. Mikami T, Ookawa K, Shimoyama T, Fukuda S, Saito H and Munakata A: KAI1, CAR, and Smad4 expression in the progression of colorectal tumor. J Gastroenterol 36: 465-469, 2001.

5. Horvath LG, Henshall SM, Kench JG, Turner JJ, Golovsky D, Brenner PC, O'Neill GF, Kooner R, Stricker PD, Grygiel JJ, et al: Loss of BMP2, Smad8, and Smad4 expression in prostate cancer progression. Prostate 59: 234-242, 2004.

6. He SM, Zhao ZW, Wang Y, Zhao JP, Wang L, Hou F and Gao GD: Reduced expression of SMAD4 in gliomas correlates with progression and survival of patients. J Exp Clin Cancer Res 30: 70, 2011.

7. van Hattem A, Brosens L, de Leng W, Morsink F, ten Kate FJ, Iacobuzio-Donahue CA, Giardiello FM and Offerhaus J: SMAD4 Protein expression in polyps of juvenile polyposis syndrome mirrors genetic status but does not reflect neoplastic progression. Gastroenterology 136: A452-A453, 2009.

8. Yan P, Klingbiel D, Saridaki Z, Ceppa P, Curto M, McKee TA, Roth A, Tejpar S, Delorenzi M, Bosman FT, et al: Reduced expression of Smad4 is associated with poor survival in colon cancer. Clin Cancer Res 22: 3037-3047, 2016.

9. Xie M, He C and Wei S: Relationship between expression of TGF- $\beta 1$, Smad2, Smad4 and prognosis of patients with resected non-small cell lung cancer. Zhongguo Fei Ai Za Zhi 18: 543-548, 2015 (In Chinese)

10. Tang ZY, Yang LY, Zhang YJ, Peng KL and Qi L: Smad4 and TGF-betal expression and clinical significance in bladder transitional cell carcinoma. Zhong Nan Da Xue Xue Bao Yi Xue Ban 31: 363-366, 2006 (In Chinese).

11. Qiu W, Schönleben F, Li X and Su GH: Disruption of transforming growth factor beta-Smad signaling pathway in head and neck squamous cell carcinoma as evidenced by mutations of SMAD2 and SMAD4. Cancer Lett 245: 163-170, 2007.

12. Miyaki M, Iijima T, Konishi M, Sakai K, Ishii A, Yasuno M, Hishima T, Koike M, Shitara N, Iwama T, et al: Higher frequency of Smad4 gene mutation in human colorectal cancer with distant metastasis. Oncogene 18: 3098-3103, 1999.

13. Blackford A, Serrano OK, Wolfgang CL, Parmigiani G, Jones S, Zhang X, Parsons DW, Lin JC, Leary RJ, Eshleman JR, et al: SMAD4 gene mutations are associated with poor prognosis in pancreatic cancer. Clin Cancer Res 15: 4674-4679, 2009.

14. Aitchison AA, Veerakumarasivam A, Vias M, Kumar R, Hamdy FC, Neal DE and Mills IG: Promoter methylation correlates with reduced Smad4 expression in advanced prostate cancer. Prostate 68: 661-674, 2008.

15. Wan M, Tang Y, Tytler EM, Lu C, Jin B, Vickers SM, Yang L, Shi $\mathrm{X}$ and Cao X: Smad4 protein stability is regulated by ubiquitin ligase SCF beta-TrCP1. J Biol Chem 279: 14484-14487, 2004

16. Wan M, Cao X, Wu Y, Bai S, Wu L, Shi X, Wang N and Cao X: Jab1 antagonizes TGF-beta signaling by inducing Smad4 degradation. EMBO Rep 3: 171-176, 2002.

17. Li L, Xin H, Xu X, Huang M, Zhang X, Chen Y, Zhang S, Fu XY and Chang Z: CHIP mediates degradation of Smad proteins and potentially regulates Smad-induced transcription. Mol Cell Biol 24: 856-864, 2004.

18. Tang Y, Katuri V, Dillner A, Mishra B, Deng CX and Mishra L: Disruption of transforming growth factor-beta signaling in ELF beta-spectrin-deficient mice. Science 299: 574-577, 2003.

19. Inman GJ, Nicolás FJ and Hill CS: Nucleocytoplasmic shuttling of Smads 2, 3, and 4 permits sensing of TGF-beta receptor activity. Mol Cell 10: 283-294, 2002.

20. Dong C, Li Z, Alvarez R Jr, Feng XH and GoldschmidtClermont PJ: Microtubule binding to Smads may regulate TGF beta activity. Mol Cell 5: 27-34, 2000

21. Wagner AD and Moehler M: Development of targeted therapies in advanced gastric cancer: Promising exploratory steps in a new era. Curr Opin Oncol 21: 381-385, 2009.

22. Powell SM, Harper JC, Hamilton SR, Robinson CR and Cummings OW: Inactivation of Smad4 in gastric carcinomas. Cancer Res 57: 4221-4224, 1997.
23. Wu DM, Zhu HX, Zhao QH, Zhang ZZ, Wang SZ, Wang ML, Gong WD, Tan $M$ and Zhang ZD: Genetic variations in the SMAD4 gene and gastric cancer susceptibility. World J Gastroenterol 16: 5635-5641, 2010 .

24. Wang LH, Kim SH, Lee JH, Choi YL, Kim YC, Park TS, Hong YC, Wu CF and Shin YK: Inactivation of SMAD4 tumor suppressor gene during gastric carcinoma progression. Clin Cancer Res 13: 102-110, 2007.

25. Calva D, Dahdaleh FS, Woodfield G, Weigel RJ, Carr JC, Chinnathambi S and Howe JR: Discovery of SMAD4 promoters, transcription factor binding sites and deletions in juvenile polyposis patients. Nucleic Acids Res 39: 5369-5378, 2011.

26. Eguchi T, Prince T, Wegiel B and Calderwood SK: Role and regulation of myeloid zinc finger protein 1 in cancer. J Cell Biochem 116: 2146-2154, 2015.

27. Tsai SJ, Hwang JM, Hsieh SC, Ying TH and Hsieh YH: Overexpression of myeloid zinc finger 1 suppresses matrix metalloproteinase-2 expression and reduces invasiveness of $\mathrm{SiHa}$ human cervical cancer cells. Biochem Biophys Res Commun 425: 462-467, 2012.

28. George SK, Vishwamitra D, Manshouri R, Shi P and Amin HM: The ALK inhibitor ASP3026 eradicates NPM-ALK ${ }^{+}$T-cell anaplastic large-cell lymphoma in vitro and in a systemic xenograft lymphoma model. Oncotarget 5: 5750-5763, 2014.

29. Vishwamitra D, Curry CV, Alkan S, Shi P and Amin HM: Sumoylation sustains the stability of NPM-ALK oncogenic protein and facilitates its nuclear accumulation in T-cell anaplastic large-cell lymphoma. Blood 124: 3586, 2014.

30. Vishwamitra D, Shi P, Wilson D, Manshouri R, Vega F, Schlette EJ and Amin HM: Expression and effects of inhibition of type I insulin-like growth factor receptor tyrosine kinase in mantle cell lymphoma. Haematologica 96: 871-880, 2011.

31. Horinaka M, Yoshida T, Tomosugi M, Yasuda S, Sowa Y and Sakai T: Myeloid zinc finger 1 mediates sulindac sulfide-induced upregulation of death receptor 5 of human colon cancer cells. Sci Rep 4: 6000, 2014

32. Chen Y, Zhang Z, Yang K, Du J, Xu Y and Liu S: Myeloid zincfinger $1(\mathrm{MZF}-1)$ suppresses prostate tumor growth through enforcing ferroportin-conducted iron egress. Oncogene 34 : 3839-3847, 2015.

33. Inoue M, Takahashi K, Niide O, Shibata M, Fukuzawa $M$ and Ra C: LDOC1, a novel MZF-1-interacting protein, induces apoptosis. FEBS Lett 579: 604-608, 2005.

34. Mudduluru G, Vajkoczy P and Allgayer H: Myeloid zinc finger 1 induces migration, invasion, and in vivo metastasis through Axl gene expression in solid cancer. Mol Cancer Res 8: 159-169, 2010.

35. Rafn B, Nielsen CF, Andersen SH, Szyniarowski P, CorcelleTermeau E, Valo E, Fehrenbacher N, Olsen CJ, Daugaard M, Egebjerg C, et al: ErbB2-driven breast cancer cell invasion depends on a complex signaling network activating myeloid zinc finger-1-dependent cathepsin B expression. Mol Cell 45: 764-776, 2012.

36. Yan QW, Reed E, Zhong XS, Thornton K, Guo Y and Yu JJ: MZF1 possesses a repressively regulatory function in ERCC1 expression. Biochem Pharmacol 71: 761-771, 2006.

37. Kundu J, Wahab SM, Kundu JK, Choi YL, Erkin OC, Lee HS, Park SG and Shin YK: Tob1 induces apoptosis and inhibits proliferation, migration and invasion of gastric cancer cells by activating Smad 4 and inhibiting $\beta$-catenin signaling. Int $J$ Oncol 41: 839-848, 2012.

38. Roth S, Laiho P, Salovaara R, Launonen V and Aaltonen LA: No SMAD4 hypermethylation in colorectal cancer. Br J Cancer 83: 1015-1019, 2000

39. Minami R, Kitazawa R, Maeda S and Kitazawa S: Analysis of 5'-flanking region of human Smad4 (DPC4) gene. Biochim Biophys Acta 1443: 182-185, 1998.

40. Schwarte-Waldhoff I and Schmiegel W: Smad4 transcriptional pathways and angiogenesis. Int J Gastrointest Cancer 31: 47-59, 2002.

41. Xia X, Wu W, Huang C, Cen G, Jiang T, Cao J, Huang K and Qiu Z: SMAD4 and its role in pancreatic cancer. Tumour Biol 36: 111-119, 2015.

42. Malkoski SP and Wang XJ: Two sides of the story? Smad4 loss in pancreatic cancer versus head-and-neck cancer. FEBS Lett 586: 1984-1992, 2012 
43. Kim SH, Lee SH, Choi YL, Wang LH, Park CK and Shin YK Extensive alteration in the expression profiles of TGFB pathway signaling components and TP53 is observed along the gastric dysplasia-carcinoma sequence. Histol Histopathol 23: 1439-1452, 2008.

44. Vishwamitra D, Curry CV, Alkan S, Song YH, Gallick GE, Kaseb AO, Shi P and Amin HM: The transcription factors Ik-1 and MZF1 downregulate IGF-IR expression in NPM-ALK ${ }^{+}$ T-cell lymphoma. Mol Cancer 14: 53, 2015.

45. Gaboli M, Kotsi PA, Gurrieri C, Cattoretti G, Ronchetti S, Cordon-Cardo C, Broxmeyer HE, Hromas R and Pandolfi PP: Mzf1 controls cell proliferation and tumorigenesis. Genes Dev 15: 1625-1630, 2001

46. Deng Y, Wang J, Wang G, Jin Y, Luo X, Xia X, Gong J and Hu J: p55PIK transcriptionally activated by MZF1 promotes colorectal cancer cell proliferation. BioMed Res Int 2013: 868131, 2013.
47. Tian X, Du H, Fu X, Li K, Li A and Zhang Y: Smad4 restoration leads to a suppression of Wnt/beta-catenin signaling activity and migration capacity in human colon carcinoma cells. Biochem Biophys Res Commun 380: 478-483, 2009.

48. Zhang B, Zhang B, Chen X, Bae S, Singh K, Washington MK and Datta PK: Loss of Smad4 in colorectal cancer induces resistance to 5-fluorouracil through activating Akt pathway. Br J Cancer 110: 946-957, 2014.

49. Jia L, Lee HS, Wu CF, Kundu J, Park SG, Kim RN, Wang LH, Erkin ÖC, Choi JS, Chae SW, et al: SMAD4 suppresses AURKAinduced metastatic phenotypes via degradation of AURKA in a TGFbeta-independent manner. Mol Cancer Res 12: 1779-1795, 2014.

50. Lee HS, Kundu J, Kim RN and Shin YK: Transducer of ERBB2.1 (TOB1) as a tumor suppressor: A mechanistic perspective. Int J Mol Sci 16: 29815-29828, 2015. 